\title{
Performare lo squat. I luoghi culturali occupati come esercizio di liveness e democrazia
}

\author{
Sara Pastore \\ Università degli studi di Napoli "Federico II"
}

\begin{abstract}
Assumendo l'intrinseca democraticità della svolta performativa delle arti - la quale eccede il principio della proprietà privata sia per l'artista che per il fruitore - il presente contributo intende esplorare le esperienze in cui la creazione dell'artista si realizza nella sua abilitazione alla creazione collettiva, mobilitando il concetto di liveness come presenza e partecipazione. II contributo, a seguito di una tipizzazione delle varie possibili declinazioni della pratica di squatting, intende restringere il focus sulla sua versione culturale, in quanto metodo performativo teso ad obiettivi estetici, comunicativi e politici. Attraverso l'interpretazione ermeneutica dei presupposti e del funzionamento di tali esperienze, nonché il riferimento - seppur sintetico, per motivi di economicità della ricerca - a casi di cultural squatting sul territorio italiano, sarà restituita una lettura di questi che li propone come momenti di attivazione e spazi d'intersezione dei diversi livelli di liveness, messi a disposizione nell'abilitazione dell'agency culturale e politica del pubblico in quanto collettività.
\end{abstract}

Assuming the democratic nature inherent to the performative turn in art practices - which exceeds the property law both for the artist and her publics - this paper aims to explore those experiences in which the artist's output enables the collective creativity, by mobilising the concept of liveness as presence and participation. The research, after an excursus about the possible categories implied in the squatting practice, intends to focus on its cultural variant, as a performative method addressed to aesthetical, communicative e political objectives. Through the hermeneutical interpretation of such experiences' premises and working, as well as the mention of some cultural squatting cases-albeit synthetic, due to the economy of the research - across the Italian territory, the paper will give back a different reading of such cases, proposing them as moment of activation and spaces of intersection of liveness' different levels, laid out to the endow cultural and political agency of publics as collectivity.

\section{Parole chiave/Key Words}

Squat art; alteristituzioni; museum studies; media studies; new institutionalism; performance art 


\section{La pratica dello squatting: definizione e classificazione}

Per il suo funzionamento, il capitalismo contemporaneo poggia su una specifica narrazione auto abilitante - che a sua volta è nutrita da e nutre complessi set di protocolli e regole per la vita quotidiana - il cui protagonista è l'individuo. Questi è infatti rappresentato come un soggetto il cui successo nella società contemporanea, soprattutto (ma non esclusivamente) in ambito lavorativo, si fonda su qualità quali l'autoimprenditorialità, la mobilità e la flessibilità. Tale discorso si basa su una circolazione di concetti di matrice individualistica e basati su un principio proprietario, che legittimano le logiche egemoniche del mercato liberista, nonché «i suoi modi di dominanti di agire la legalità e all'azione di stato» ${ }^{1}$. Ciò è funzionale a non lasciar trasparire alcuno spazio di sfida o di elaborazione alternativa all'attuale sistema capitalista, al punto che, nelle parole di Fisher «è più facile immaginare la fine del mondo che la fine del capitalismo»².

Allo stesso modo, la norma giuridica contemporanea propone come sole opzioni praticabili la forma pubblica o quella privata. Secondo la linea di ricerca legata al movimento dei commons, di contro, esisterebbe una terza categoria, vale a dire quella del comune. Esso corrisponderebbe alla ricchezza comune del mondo materiale ma anche di tutto ciò che si ricava dalla produzione sociale e che è a sua volta messo a disposizione per l'interazione sociale. Una prima caratteristica del comune è quella, dunque, di essere nettamente distinto non solo rispetto al privato, ma anche rispetto al pubblico. L'occultazione del comune attraverso la narrazione binaria pubblico/privato corrisponderebbe così alla prima tattica della sua sottrazione, poiché «ogni processo di privatizzazione deciso dall'autorità politica espropria ciascun cittadino della sua quota parte del bene comune sottratto, proprio come avviene nel caso dell'espropriazione di un bene privato» ${ }^{3}$. Mentre però, sulla base della tradizione costituzionale liberale, in quest'ultimo caso il proprietario privato è tutelato dinanzi l'autorità pubblica, ciò non viene applicato nel caso dei beni comuni, vale a dire i beni della collettività, per i quali non esiste alcuna tutela giuridica.

\footnotetext{
${ }^{1}$ D. Harvey, The Right to The City, in «New Left Review», vol. 53, 2008.

2 M. Fisher, Realismo Capitalista, Nero Edizioni, Milano 2018.

${ }^{3}$ U. Mattei, Beni Comuni. Un Manifesto, Laterza, Bari 2011, p. 5.
} 
II caso della città appare qui emblematico poiché essa, in questa prospettiva, si configura come un inesauribile serbatoio del comune in cui confluiscono elementi materiali e immateriali, i quali costituiscono una ricchezza intensivamente prodotta ed estensivamente disponibile, ma anche sistematicamente catturata, controllata, sfruttata e privatizzata. Si parla a questo titolo di «diritto alla città " $^{4}$ : esso si riferisce non solo all'accesso alle risorse, ma anche e soprattutto all'esercizio di un potere collettivo sui processi di urbanizzazione. L'urbanizzazione, però, non va inquadrata solo come trasformazione infrastrutturale: essa implica infatti la costruzione di un asset di valori, criteri e pratiche quotidiane che a loro volta confluiscono in specifici modelli di persona urbana, e infondono forza a narrazioni tessute ad hoc per corroborarli. Quello che si viene a configurare è dunque

\begin{abstract}
un mondo in cui l'etica neoliberista di un intenso individualismo possessivo e la sua complementare rinuncia al supporto per le forme di azione collettiva possono diventare il template per la socializzazione interpersonale (...). I risultati sono indelebilmente inscritti nelle forme spaziali delle nostre città, che diventano sempre di più città di frammenti fortificati, di gated communities e di spazi pubblici privatizzati tenuti sotto sorveglianza costante. ${ }^{5}$
\end{abstract}

Alcune esperienze contemporanee di riappropriazione dello spazio possono essere rilette alla luce di queste considerazioni: affrontato nella sua dimensione strutturale, infrastrutturale e sovrastrutturale, il cultural squatting rappresenta un'innovativa metodologia dell'urbanizzazione, all'insegna della democraticità e della partecipazione.

A partire dagli ultimi decenni la comunità accademica internazionale ha dedicato numerose linee di ricerca alle pratiche di squat osservandone distribuzione, funzionamento, modelli e impatto. Nel quadro del presente contributo, ci si è serviti in particolare della tipizzazione elaborata da Prujit ${ }^{6}$ al fine di operare una divisione in categorie che, nonostante si serva di una necessaria semplificazione, risulta utile alla contestualizzazione dei fenomeni che di qui a poco si intende approfondire. Nella sua ricerca, la cui base empirica è costituita dall'esperienza di squatting nei Paesi Bassi, nel Regno Unito, in Germania e in Italia, l'urban squatting è definito nei termini di appropriazione e utilizzo di un'abitazione o un edificio di altro tipo senza il consenso del proprietario. Nel caso dell'urban squatting inteso come mo-

\footnotetext{
${ }^{4}$ D. Harvey, op. cit.

${ }^{5}$ Ibidem.

${ }^{6}$ H. Prujit, The Logic of Urban Squatting, in «International Journal of Urban and Regional Research», vol. 37, n. 1, 2011.
} 
vimento sociale, che rileva all'interno dello spazio urbano e periurbano una geografia di spazi in disuso come potenziali risorse, esso è soggetto a numerose interpretazioni e chiavi di lettura, offrendosi alla ricerca come manifestazione di sfida al sistema economico dominante, come espressione di dissenso politico, come pratica culturale o, ancora, come soluzione materialmente immediata ad un'esigenza altrettanto materiale ed urgente. Accettando la diversità come suo punto di partenza, quella delineata da Prujit è dunque un'analisi comparativa delle tipologie di squatting che, con il sostegno della teoria dei New Social Movement ${ }^{7}$ da cui prende in parte le mosse, osserva i movimenti sociali contemporanei nei termini strutturali di un network e in quelli organizzativi di una piattaforma orizzontale - nonché ne considera in maniera non subordinata obiettivi politici, economici e culturali.

Le configurazioni sono dunque cinque: lo squatting da privazione; lo squatting come strategia di housing alternativa; lo squatting imprenditoriale; lo squatting conservativo; lo squatting politico. Come si vedrà, si tratta di configurazioni ricavate dalla combinazione di caratteristiche e, essendo modelli tipici e tipizzanti essi non esauriscono tutte le specifiche realizzazioni effettive del fenomeno, che piuttosto si pongono di volta in volta sul continuum che tali modelli tracciano.

Per squatting da privazione si intende quel tipo di occupazione, generalmente abitativa, da parte di individui socio-demograficamente ed economicamente svantaggiati: è caratterizzato generalmente da un'organizzazione definita e una divisione di ruoli marcata tra abitanti ed attivisti. A scopo abitativo è anche la seconda categoria dove l'occupazione diventa il modo di conquistare una condizione abitativa alternativa, spesso temporanea e dovuta a necessità contingenti, non per forza di natura economica - ma anche ad esempio culturale, con riferimento ad altre forme di socializzazione e modelli comunitari, o a pratiche dell'ambito DIY (Do It Yourself).

La terza configurazione, quella che Prujit definisce come entrepreneurial squat$t_{i n g}{ }^{8}$, non risponde invece - almeno non direttamente - ad un'emergenza abitativa: progetti di beneficienza, spazi di lavoro, cinema, scuole alternative, laboratori, spazi espositivi e così via, sono solo alcuni delle possibili realizzazioni. Per entrepreneurial squatting,

\footnotetext{
${ }^{7}$ A. Melucci, New Social Movements. A theoretical Approach, in «Social Science Information», vol. 19, n. 2, 1980.

${ }^{8} \mathrm{Si}$ è scelto, qui e in tutto il testo, di mantenere la locuzione originale in inglese, poiché il termine corrispondente italiano ("imprenditoriale") restituisce, come si vedrà in seguito, solo parzialmente il significato inteso.
} 
dunque, si intendono quei casi in cui la pratica di squatting restituisce opportunità per un ampio range di organizzazioni, offrendo opzioni alternative al reperimento delle risorse e alla realizzazione delle attività. Seppure possa sembrare fuorviante, la nozione di imprenditorialità si riferisce in questo contesto ad un approccio in cui individui o gruppi sviluppano, implementano e sostengono soluzioni a problemi di ordine sociale, culturale o ambientale; sono ivi comprese dunque anche le organizzazioni non profit o blended, in cui il principale outcome è definito in termini di "ritorno sociale" 9 .

In Italia, ad esempio, questi spazi corrispondono secondo Prujit ai Centri Sociali, e tracciano una geografia di esperienze numerose e assai diverse tra loro, nonché protagoniste di importanti funzioni culturali, politiche e identitarie.

Ancora, lo squatting può assurgere a tattica di preservazione di un landscape cittadino o naturale: è il caso della quarta tipologia, quella dello squatting conservativo. Esso in genere segue alla pianificazione a all'annuncio di una trasformazione, spesso divulgata tramite un discorso pubblico che combina riqualificazione, sviluppo e valorizzazione, di luoghi dal valore storico, culturale e sociale. Per via del suo obiettivo è spesso una soluzione temporanea accompagnata da manifestazioni e richieste ufficiali.

Infine, abbiamo lo squatting politico, in cui l'occupazione non è di per sé un obiettivo quanto piuttosto «un potenziale strumento di confrontazione dello stato» ${ }^{10}$. Lungi da essere l'unica forma di squatting che implica una riflessione politica (che è invece sottesa in ogni declinazione della pratica) lo squatting politico trova in quest'ultima il suo perno strategico, programmatico ed esplicito.

I luoghi culturali occupati contemporanei, che rivisitano la proposta dei Centri Sociali della fine del secolo scorso, intersecano nelle loro proprietà le varie configurazioni: essi però sono soprattutto caratterizzati dal ritorno sociale della loro attività e, interpolando la vocazione organizzativo-culturale con obiettivi di carattere civico, riconsegnano ai propri contesti urbani una specifica versione dell'imprenditorialità.

Le versioni attuali dei Centri Sociali possono venire a rappresentare, così, un esempio di quelle comunità informali che costituiscono una significativa componente dell'«internal ar-

\footnotetext{
${ }^{9}$ J.L. Thompson, The World of the Social Entrepreneur, in «International Journal of Public Sector Management», vol. 15, n. 5, 2002.

${ }^{10}$ H. Prujit, op. cit., p. 20.
} 
rangement ${ }^{11}$ dell'economia delle città contemporanee: queste sono viste come eco-sistemi locali che riescono a creare i propri ambienti istituzionali tramite l'attivazione e la messa a disposizione delle risorse nascoste del territorio. In questo senso, i Centri Sociali

[...] Seppure già noti quale modalità di aggregazione politica tra gruppi di azione locali, stanno assumendo un peso crescente, anche come comunità creative e del cambiamento. [...] Comunità informali, luoghi creativi e drivers di produttività urbana ${ }^{12}$.

Rispetto ai loro predecessori essi si pongono, sull'onda da un lato della delusione storica dei movimenti sociali sessantottini, dall'altro su quella dell'incontro con i nuovi movimenti sociali globali, in una posizione di maggiore dinamicità e flessibilità politica rispetto ai loro predecessori, abilitata da una maggiore e più centrale vocazione culturale e risultante in alcune caratteristiche. Dal punto di vista strettamente politico essi non corrispondono a fazioni stabili, rinunciando spesso ad un'auto-classificazione identitaria e preferendo a quest'ultima una riflessione più informale, ma anche più integrata, che mette in funzione i concetti di decolonizzazione, cittadinanza universale ed attivazione sociale. Dal punto di vista culturale, e delle politiche culturali, di contro, la loro posizione è molto più marcata e programmatica. Essa è spesso legata ai criteri attinenti all'ambito del cultural open source - che costituisce una risposta all'ipermercificazione della cultura da un lato, e alla cattura della controcultura dall'altro, ad opera sia del mercato che del potere politico - e del DIY educativo e formativo, mirando a costituire il movimento come network di conoscenze e saperi condivisi, trasmessi e scambiati in maniera non tradizionale.

La vocazione culturale è dunque centrale ed è a partire da questa che si diramano le varie riflessioni sociali, politiche ed economiche: assumendo la produzione e il consumo creativo come elementi del "repertorio d'azione"13 dei movimenti sociali, il cultural squatting è in un certo senso figlio del contesto capitalista cognitivo contemporaneo, poiché fornisce una risposta ai suoi tentativi di sussunzione, e implica il riconoscimento delle pratiche mediali e delle rappresentazioni culturali come territori di contesa sociale. Non a ca-

\footnotetext{
11 M.P. Vittoria, P. Napolitano, Comunità informali come luoghi creativi e drivers di produttività urbana. II caso dei Centri Sociali a Napoli, in «Rivista economica del Mezzogiorno», Fascicolo 1-2, 2017.

12 Ibidem.

${ }^{13}$ C. Tilly, Contentious Repertoires in Great Britain, 1758-1834, in «Social Science History», vol. 17, n. 2, 1993.
} 
so, e maggior ragione posizionandosi nell'impervio terreno di riflessione dell'arte nello spazio metropolitano, e dunque pubblico, esso può assumere direzioni e sfumature ambigue e spesso in antitesi tra loro. Nonostante ciò, in alcuni casi qui considerati, rimangono aperte linee di fuga e di reinvenzione dei format e dei codici artistici e civici.

\title{
Arte pubblica e spazio pubblico: il caso del cultural squatting
}

II termine public art viene oggi utilizzato per indicare i movimenti e le esperienze artistiche che si caratterizzano per «una dimensione spaziale e comunicativa aperta, ponendosi fuori dalle riserve protette di musei, gallerie e simili ambienti privilegiati» ${ }^{14}$. A partire dai presupposti di comunicazione diretta tra artisti e pubblico, ripensamento degli spazi urbani e del ruolo dell'arte, queste pratiche indagano la natura dell'opera d'arte e quella dello spazio pubblico. È infatti nella loro relazione reciproca che i due termini ritrovano la possibilità di una mutua espansione: la prima diventa pratica partecipata, il secondo, da una connotazione topografica e architettonica, ne assume una interazionale.

A partire dagli anni '90, con la differenziazione ulteriore di strumenti, tecniche e pubblici, nonché con l'emersione di esperienze innovatrici influenzate dalle metodologie inaugurate dall'Institutional Critique ${ }^{15}$ e dall'estetica relazionale ${ }^{16}$, è possibile parlare di new genre public art - espressione che assume come centrale la triangolazione tra artisti, pubblici e comunità, in una dimensione di senso contrario rispetto a quella delineata dai luoghi deputati atti a selezionare e isolare l'opera dal mondo:

\begin{abstract}
Scegliere di lavorare fuori dai luoghi espositivi significa lavorare nel contesto urbano come luogo delle condizioni concrete di vita e anche sulle forme di interazione sociale fra le persone, fra cui l'artista si colloca, in un rapporto orizzontale di collaborazione ${ }^{17}$.
\end{abstract}

Così, la riconcettualizzazione dei pubblici implica necessariamente quella dello spazio pubblico come spazio sociale: posizionandosi fuori dai confini della galleria, l'opera d'arte pubblica si distingue poiché entra in uno spazio che è vivo, instaurando un dialogo

\footnotetext{
${ }^{14}$ L. Perelli, Public Art. Arte. Interazione e progetto urbano, FrancoAngeli, Milano 2015, p. 9.

${ }^{15} \mathrm{G}$. Raunig, G. Ray (a cura di), Art and contemporary critical practice: reinventing institutional critique, MayFly Books, London 2009.

${ }^{16}$ N. Bourriad, Estetica Relazionale, PostMedia Books, Milano 2010.

${ }^{17}$ L. Perelli, op. cit., p. 53.
} 
con l'intreccio di istanze individuali e collettive, questioni sociali, eventi politici e significati culturali. II ruolo sociale dell'arte si fa più complesso nel contesto dell'arte pubblica poiché, se da un lato essa si pone come elemento che promuove l'accesso agli spazi pubblici, dall'altro lato implica anche una problematizzazione di tale accesso, evidenziando le dinamiche di esclusione ed inclusione dei luoghi. Ciò però non va inteso nel senso di un'estetizzazione metropolitana: lo spazio urbano e periurbano, difatti, subisce tali interventi secondo un'ottica partecipativa che non ne rinnega ma anzi ne rafforza l'ospitalità, proponendo una versione di arte nello spazio pubblico che si discosta dai processi di museificazione e gentrificazione e dai relativi modelli di città museo, città d'arte, città creativa e così via.

La pratica artistica emerge in tal modo come prassi civica, predisponendo le condizioni necessarie per l'attualizzazione di quella dimensione politica che è inevitabilmente insita nelle azioni creative. Essa è così «azione artistica che interviene nelle situazioni sociali concrete in un territorio dato»; "lavoro artistico nei territori urbani di confine e marginalità»; «pratica di inclusione», «pratica di condivisione» e «costruzione di socialità» ${ }^{18}$.

Progressivamente, l'ecosistema artistico si rende disponibile ad accogliere un numero sempre maggiore di esempi che intendono la produzione culturale come azione politica, legando arte e movimenti sociali attraverso una traduzione di competenze, risorse e immaginari dalle forme artistiche a quelle organizzative e viceversa.

In questa prospettiva, la natura collettiva di queste pratiche ritrova una ulteriore ragion d'essere nell'intrinseco legame tra la struttura organizzativa a network e la cultura multiforme, plurale e rizomatica dei luoghi urbani contemporanei. I primi anni 2000 che sono gli anni delle grandi manifestazioni contro la corporate globalization nel clima mediale di espansione e intensificazione dell'interattività - sono stati infatti fondamentali per lo sviluppo dell'attivismo creativo. In particolare, ciò è vero per quelle sue forme che implicavano le pratiche collettive e collaborative e il coinvolgimento civico, abilitato da una rete comunicativa sempre più intensa e globale.

Il meccanismo reticolare e capillare che ha rinnovato i movimenti sociali ha in qualche modo toccato anche la pratica artistica, i cui vecchi parametri sembravano gradual-

\footnotetext{
${ }^{18}$ L. Perelli, op. cit., p. 64.
} 
mente venir meno. Ciò ha determinato un'espansione delle modalità del fare arte lungo direzioni inedite, con la combinazione di riflessioni e tecniche della teoria politica, dell'attivismo, della community art, del teatro e della performance art, nonché con la riemersione di un'arte politica seriamente coinvolta.

A cavallo tra i due secoli, dunque, si è diffusa - a partire dal mondo anglosassone per poi estendersi ai maggiori poli della produzione artistica occidentale - questa dinamica il cui effetto di rinnovamento ha agito dentro e fuori il sistema dell'arte, dimostrando prepotentemente la permeabilità tra questo e le altre sfere del sociale:

Ciò che le istituzioni non avrebbero permesso, ciò che non potevano o volevano accogliere tra le loro mura, gli artisti lo facevano al di fuori, generando così una costante pressione omeostatica di ritorno sulle istituzioni stesse. Sembrava essere una rivoluzione in slow-motion, un'evoluzione sociale necessaria che gli artisti contribuivano a progettare e modellare ${ }^{19}$.

Diventa evidente, in questo modo, il ruolo fondamentale giocato dall'arte nei confronti dei movimenti radicali minoritari, soprattutto nell'apertura ad un bacino di partecipazione più ampio, al di fuori delle sottoculture politiche.

Le rispettive vulnerabilità della produzione controculturale e dei movimenti sociali, difatti, vengono mitigate attraverso una programmatica e mai subalterna relazione tra i due termini, realizzando un'agenda sociale per l'arte oltre il contenuto estetico politicizzato e amplificando la portata della comunicazione e degli immaginari di movimento. La cultura dei movimenti sociali, dunque, da un lato allarga la cornice della produzione controculturale oltre quella del materiale grafico e politico, risaltando le formazioni sociali create dai movimenti e le relazioni che essi instaurano con le tecnologie della comunicazione e della creatività; dall'altro, avanza la concezione dell'artista come agente di cambiamento. Umanizzando, naturalizzando e rendendo comprensibili le situazioni politiche, l'arte risulta avere un ruolo fondamentale - specialmente nel quadro della netta polarizzazione tipica dei conflitti.

Le tecniche e le tattiche creative della protesta basata sul networking che si diffondono all'inizio del XXI secolo provengono infatti in gran parte dall'armamentario cultura-

${ }^{19}$ A. Moore, Occupation Culture: Art \& Squatting in the City from Below, Autonomedia, Brooklyn 2015, p. 100. 
le del movimento di squatting politico e sono basati sulla congiunzione tra le skills relative all'azione culturale e quelle relative all'azione diretta, con l'obiettivo di infondere l'arte e la cultura nei movimenti popolari per creare un nuovo linguaggio e nuove forme della resistenza, in linea con la naturale inclinazione cerimoniale e creativa della manifestazione. L'atto dell'occupazione, in tutte queste esperienze, svolge un ruolo fondamentale, poiché rompe lo status quo incidendo sul livello spaziale e territoriale - nonché su quello logico - e assumendo un valore sia di mercato sia simbolico, poiché produce un disturbo materiale e narrativo inceppando gli ingranaggi della macchina semantica e normativa. Parallelamente, la pratica artistica si impone come elemento della vita quotidiana incidendo sull'immaginario urbano e sulle logiche territoriali.

La relazione tra arte e squat, dunque, pur mantenendo una certa quota di problematicità, poiché getta un ponte tra l'artworld (il circuito di spazi espositivi, accademie, outlet e organizzazioni istituzionali, che sono legati alle culture dominanti, ai mercati tradizionali e ai governi ufficiali) e l'universo dello squatting (che è invece sottoculturale) è spiegabile in termini strutturali (economici) e sovrastrutturali (ontologici). Dal punto di vista economico, infatti, nonostante oggi l'arte sia profondamente imbricata nelle dinamiche di un mercato capitalista e neoliberista, la professione artistica per sua natura con concorda bene con questo sistema, che lascia come uniche opzioni praticabili quelle dell' «artista imprenditore del sé e quello a carico del welfare ${ }^{20}$. Solo in rari e spettacolarizzati casi presa sul serio dal punto di vista professionale, e più spesso ridotta all'ambito del lusso e del loisir, la pratica artistica risulta, a partire dalla stessa formazione, generalmente non sostenibile, in un circolo vizioso che promette di mantenerla sistemicamente elitaria. A ciò si aggiunge inoltre una lettura che ritrova una effettiva parentela tra arte e squat, la quale emerge sotto forma di somiglianza su vari piani: nel lavoro richiesto agli artisti e agli attivisti; nella temporalità frequentemente limitata dell'esposizione e dell'occupazione; nella combinazione di funzione sociale e disposizione simbolica: così, l'occupazione può essere considerata non solo come tattica politica, ma anche come forma di pratica estetica.

${ }^{20}$ M. Baravalle, Alteristituzioni e Arte. Tra Governamentalità e Autonomia, in «OperaViva Magazine», Agosto 2018. 
Oltre, dunque, a restituire un'ampia gamma di forme di ospitalità per l'arte, supportando forme altrove inospitabili e incubando movimenti produttivi e modi d'uso innovativi, la stessa azione di squatting è in sé un fatto culturale e un atto performativo, che si avvale di elementi dello spettacolo e della creatività. Questi spazi diventano così veri e propri bastioni della cultura radicale, che annunciano la loro presenza fiancheggiando le mura dei quartieri che abitano con striscioni, graffiti e poster, e restituiscono lo spazio urbano un campo di battaglia politica e un territorio performativo.

\section{Attraverso la lente della liveness: un dispositivo concettuale per la lettura del cultural squatting}

Sono sempre di più, a livello nazionale e non solo, le esperienze per cui la pratica del cultural squatting costituisce la stessa condizione d'esistenza. Queste ridisegnano la cartografia dell'arte e della cultura urbana e riscrivono le regole della produzione e della fruizione culturale attraverso le linee di ricerca della diffusione libera, delle economie alternative e della messa in condivisione di spazi e risorse. Allo stesso tempo, queste stesse prospettive ritornano sotto forma di ulteriori forme dell'azione diretta a disposizione del repertorio dei movimenti, aprendo nuove possibilità di comunicazione e socializzazione grazie alle specifiche competenze dell'arte e della performance.

Penso al S.a.L.e. Docks di Venezia, il Macao di Milano, il Maam di Roma, L'Asilo a Napo$\mathrm{li}^{21}$, che sono solo alcuni dei molti esempi che, ponendosi come risultati di una ricerca artistica e politica in atto dagli anni '60, e ancor più sull'onda della spinta del movimento dei beni comuni dello scorso decennio, aprono nuove linee di ricerca nella metodologia non solo della produzione e della fruizione artistica, ma anche del vivere quotidiano e dell'agire sociale.

Nell'introdurre una dinamica esperienziale, sia per l'artista che per il fruitore, che trascende il principio della proprietà privata ${ }^{22}$, è possibile rilevare un certo potenziale di democraticità nella svolta performativa delle arti tardonovecentesca. Le esperienze di

\footnotetext{
${ }^{21}$ Per ulteriori approfondimenti dei casi studio di riferimento, qui trattati brevemente per l'economicità della ricerca, si rimanda ai siti web di riferimento e alle pagine social:

Macao: https://www.macaomilano.org/; https://www.facebook.com/macaomilano;

S.a.L.E. Docks: http://www.saledocks.org/; https://www.facebook.com/saledocks;

L’Asilo: http://www.exasilofilangieri.it/; https://www.facebook.com/lasilo;

Maam: https://www.facebook.com/museoMAAM/

${ }^{22}$ A.B. Oliva, Territorio Magico. Comportamenti alternativi nell'arte, Le Lettere, Firenze 2002.
} 
cultural squatting si propongono come ulteriore momento di questo percorso, poiché continuano la trasgressione dello spazio rinascimentale tramite l'appropriazione di quello collettivo. Tale tragitto culmina inoltre con l'occupazione degli spazi digitali, a causa dell'attuale impossibilità di realizzare un discorso pubblico al di fuori delle logiche della mediatizzazione e ancor più della trans-mediatizzazione come dinamica comunicativa privilegiata del mediascape contemporaneo. Esse, inoltre, se da un lato si discostano dall'idea del sociale come immanent togertheness, implicando necessariamente una qualche quota di conflittualità, dall'altro sono anche al sicuro dal rischio di incorrere nel populismo artistico, non relegando l'antagonismo allo spazio autonomo dell'arte e assurgendo, così, a opzione virtuosa in alternativa alle due derive rarefatte delle pratiche culturali performativo-relazionali ${ }^{23}$.

Intendendo la liveness come una relazione specifica con l'alterità o una precisa modalità di coinvolgimento, essa è tra le proprietà di quelle pratiche culturali che, attraverso un metodo di ricerca azione, mobilitano forze e risorse di ambiti di competenza diversi per ripensare il fare arte e lo stare nella società.

Se l'artista detiene un'urgenza politica, infatti, questa non è quella di restituire rappresentazioni, quanto piuttosto di investigare i processi e gli apparati che controllano queste ultime: si tratta di un allontanamento programmatico dall'ossessione nei confronti del contenuto o del tema, attraverso cui è possibile realizzare il necessario salto dal teatro politico alla politica del teatro.

I corpi e le azioni dei performer organizzano una serie di riflessioni che intersecano gli assi della soggettività, del luogo, della mercificazione, del controllo, della convenzione e della politica. Chi si esprime e verso chi; qual è il contesto spaziale dell'espressione e che valore esso può determinare; chi detiene il controllo nella produzione di significati e in che modo lo esercita; quali sono le posizioni politiche e sociali necessariamente implicate - contestate o rafforzate - nella pratica artistica. E le stesse questioni, si ritrovano appunto nelle esperienze di cultural squatting.

In particolare, innestandosi sul nucleo di riflessione della public art, il cultural squatting rappresenta una pratica che in maniera iterativa - poiché lo fa sia nella sua ge-

\footnotetext{
${ }^{23}$ M. Baravalle, L'Autunno Caldo del Curatore. Arte, neoliberismo e pandemia, Marsilio, Venezia 2021.
} 
nesi che nella sua successiva offerta civica e sociale - approfondisce ulteriormente il ripensamento di individui, collettività e habitat, assunti nella loro triangolazione.

In questi casi, la creazione dell'artista si realizza nella sua abilitazione della creazione collettiva: la performance è qui intesa non come la predisposizione e l'esecuzione dello spettacolo, quanto piuttosto come fabbricazione di una piattaforma organica, dinamica, sensibile e abitabile.

La questione dello spettatore è di particolare importanza, poiché costituisce il fulcro della discussione riguardo la relazione tra arte e politica. È ad esempio ciò che intende Ranciére ${ }^{24}$ quando invoca la necessità di un teatro senza spettatori: non un teatro le cui poltrone sono vuote, ma un teatro in cui l'atto spettatoriale non implichi una qualche passività; un teatro in cui, piuttosto che come voyeur passivi, i fruitori si configurino come partecipanti attivi. L'inizio del processo di emancipazione dello spettatore risiederebbe così nella sfida all'opposizione tra vedere e agire. Ciò permetterebbe l'emersione delle comunità estetiche e creative come potenziali comunità del dissenso, realizzando una disposizione insita della performatività, di cui il teatro è modalità comunicativa per eccellenza. Lo sviluppo di quest'ultimo, infatti, è esso stesso legato alla presenza di una comunità locale di cui esso è una costituzione estetica: vale a dire, un «modo di occupare uno spazio materiale e cronologico [...] che precede e preforma le leggi e le istituzioni politiche ${ }^{25}$.

Intesa non come caratteristica o effetto di un oggetto artistico, ma piuttosto secondo un'accezione mobile e fenomenologica, emerge una rivisitazione del concetto di liveness che permette di osservarla come una forma che si basa sull'accettazione di un nuovo patto spettatoriale, «che riarticola l'hic et nunc nella continuità tra offline e online ${ }^{26}$. In questo senso la liveness agisce producendo una posizione del soggetto, la quale è costruita in maniera relazionale rispetto agli altri soggetti del pubblico, i contesti, le istituzioni e le tecnologie.

In quanto azione costruita e socializzante, «teatro da organizzare, produrre e performare ${ }^{27}$, i casi di cultural squatting sono leggibili attraverso la prospettiva dell'evento. Nei termini di Scannell l'evento innesca il mutamento storico trasformando

\footnotetext{
${ }^{24}$ J. Ranciére, The Emancipated Spectator, Verso Books, London \& New York 2009.

${ }^{25}$ Ivi, p. 6.

${ }^{26}$ L. Gemini, S. Brilli, F. Giuliani, I/ dispositivo teatrale alla prova del Covid-19. Mediatizzazione, liveness e pubblici, in «Mediascapes Journal», n. 15, 2020, pp. 44-58.

${ }^{27}$ P. Scannell, Authenticity and Experience in «Discourse Studies», vol. 3, n. 4, 2001, pp. 405-411.
} 
le stesse categorie culturali che plasmano e limitano l'azione umana influenzando così, almeno in potenza, la causalità sociale. Le esperienze di cultural squatting, al crocevia tra la pratica artistica e quella di movimento, si caratterizzano come forma peculiare di eventful protest - dimostrazioni pubbliche, azioni dirette o anche iniziative simboliche caratteristiche dei movimenti dell'ultimo ventennio ${ }^{28}$.

L'eventful protest è in grado, dunque, di incidere sulle strutture preesistenti alimentando i meccanismi del cambiamento sociale, attraverso lo sviluppo di network, framework culturali e relazioni interpersonali. In questo senso,

[...] Gli eventi di protesta [...] costituiscono processi durante i quali l'esperienza collettiva si sviluppa nelle interazioni di diversi attori individuali e collettivi, che con ruoli e scopi differenti vi prendono parte $\left[\ldots . .{ }^{29}\right.$

Nella prospettiva dell'eventful protest, sia come pratica artistica che di movimento, i luoghi culturali occupati assumono un valore strumentale (sono risorse a disposizione di un determinato gruppo con obiettivi specifici) sia un effetto socializzante: essi, infatti costruiscono identità, producono relazione interne, facilitano la comunicazione esterna e costruiscono narrazioni, drammaturgie e rituali che permettono alle "persone di farsi comunità». ${ }^{30}$

È così che è possibile leggere le esperienze di cultural squatting in termini di liveness, di cui realizzano, intersecandoli, i vari livelli: co-presenza fisica di performers e audience, simultaneità temporale di produzione e ricezione, istantaneità dell'esperienza; possibilità di ripetizione infinita tramite la registrazione; senso di co-presenza e connessione tra partecipanti (sia online che offline). Questi sono infatti combinati e attivati nelle varie fasi che accompagnano e costituiscono le azioni collettive di riappropriazione urbana.

Tale processo comincia con la narrativizzazione mitopoietica dell'occupazione che inaugura un doppio dello spazio materiale sul piano dell'immaginario. È il caso ad esempio di Macao, il Nuovo Centro per le Arti, la Cultura e la Ricerca, il cui debutto nel landscape urbano milanese avviene con l'occupazione di Torre Galfa, preceduta da una

\footnotetext{
${ }^{28}$ D. Della Porta, Eventful Protest, Global Conflicts: Social Mechanisms in the Reproduction of Protest, in J. Jasper, J. Goodwin (a cura di), Contention in Context: Political opportunities and the emergence of protest, Stanford University Press, Stanford 2011, pp. 256-276.

${ }^{29}$ Ibidem.

${ }^{30}$ Ibidem.
} 
intensa campagna comunicativa che si dipana sia sul piano virtuale che su quello fisico, intrecciandone traiettorie, tecniche e strumenti. L'occupazione di Torre Galfa, seppur temporanea (l'edificio fu infatti sgomberato pochi giorni dopo) fu preceduta e accompagnata da una campagna video su YouTube, una open call lanciata sul neonato sito del Centro, interviste condotte nei vari quartieri della città, un ciclo di assemblee e seminari e una chiamata finale al punto di raccolta gestita tramite mailing list (strumento relativamente obsoleto il cui utilizzo è riconducibile sia alla sua portata simbolica legata al milieu dei centri sociali sia a questioni di privacy e sicurezza). In questo modo l'occupazione stessa costituisce il primo progetto culturale di Macao, che nella sua declinazione estetica (e dunque politica) ha abilitato e canalizzato l'agency di centinaia di cittadini e cittadine, costruendo attraverso la performance una rete di soggettività collaborative.

Tale progetto può continuare, inoltre, con la costituzione di spazi viventi in virtù delle stesse comunità che accolgono, coniugando esigenze abitative e desideri comunicativi: emblematico in questo senso è il caso del Maam (Museo dell'Altro e dell'Altrove Metropoliz) che si configura appunto come un museo abitato e vivo in cui il rapporto tra curatori, ospiti, pubblici e artworks si muove trasversalmente lungo i vari assi della liveness.

Le esperienze di cultural squatting, dunque - di cui si è potuto in questa sede restituire solo alcuni riferimenti a titolo esemplificativo, volendo privilegiarne una proposta d'interpretazione - attuano, attraverso campagne on/off che non stentano a riconoscere la porosità del territorio virtuale, una costruzione dell'evento e del suo senso tramite la sinergia dei vocabolari e dei repertori dell'online e dell'offline, risultanti nell'elaborazione di un nuovo linguaggio performativo. Quest'ultimo produce un riverbero che oltrepassa i confini del sistema dell'arte, infrangendoli, e implicando nuove modalità della creatività, della militanza, nonché della soggettivazione politica e della socializzazione collettiva.

Se da un lato i due costituenti chiave del cultural squatting - la performatività e l'intervento estetico nello spazio urbano - sono device espressivi non del tutto estranei ai meccanismi di appropriazione neoliberali, la particolare interpretazione che esso ne restituisce innesca un'esperienza per i soggetti coinvolti definibile in termini di "autenticità" ${ }^{31}$ che trascende e in realtà disattiva una serie di opposizioni binarie: arte/quotidiano, auto-

\footnotetext{
${ }^{31}$ P. Scannell, op. cit.
} 
re/pubblico, azione/rappresentazione, soggettivo/collettivo, live/mediatizzato - e così via. A ciò si aggiunge l'introduzione di una diversa temporalità, quella dell'evento, che fornisce una lente di lettura sia delle pratiche specifiche, sia delle trasformazioni strutturali del cosmo sociale e culturale su cui si innestano.

Così, la pratica di squatting, nella sua dimensione istantanea - l'ingresso, la sovversione e la riappropriazione materiale dello spazio - sia in quella processuale - la messa a disposizione dello spazio come luogo del comune - si propone come esercizio di liveness e democrazia, smascherando l'intrinseco legame fra questi.

Enfatizzando il composto, eterogeneo, aperto e «indeterminato carattere della tradizione democratica» $^{32}$ (si potrebbe dunque anche dire performativo), il cultural squatting assurge così a potenziale strumento atto a disporre le soggettività all'interno di una matrice democratica.

\section{Riferimenti Bibliografici}

P. Auslander, From Acting to Performance. Essays on modernism and postmodernism, Routledge, London \& New York 1997.

P. Auslander, Liveness. Performance in a Mediatized Culture, Routledge, London \& New York 2009.

M. Baravalle, Alteristituzioni e Arte. Tra Governamentalità e Autonomia, in "OperaViva Magazine», agosto 2018 (ultimo accesso 23/11/2021).

M. Baravalle, L'Autunno Caldo del Curatore. Arte, neoliberismo e pandemia, Marsilio, Venezia 2021.

T. Bazzichelli, Networking. La rete come arte, Costa \& Nolan, Genova 2006.

A. Bonito Oliva, Il territorio magico. Comportamenti alternativi nell'arte, Le Lettere, Firenze 2009.

N. Bourriad, Estetica Relazionale, PostMedia Books, Milano 2010.

P. Carmona, T. Herreros, R. Sánchez Cedillo, N. Sguiglia, Social centres: monsters and political machines for a new generation of movement institutions, in "Transversal text», vol. 4, 2008, (ultimo accesso 23/11/2021).

D. Della Porta, Eventful Protest, Global Conflicts: Social Mechanisms in the Reproduction of Protest, in J. Jasper, J. Goodwin (a cura di), Contention in Context: Political opportunities and the emergence of protest, Stanford University Press, Stanford 2011, pp. 256-276.

${ }^{32}$ C. Mouffe, P. Holdengräber, Radical Democracy: Modern or Postmodern?, in «Social Text», vol. 21, 1989 , p. 41. 
M. Fisher, Realismo Capitalista, Nero Edizioni, Milano 2018.

L. Gemini, S. Brilli, F. Giuliani, Il dispositivo teatrale alla prova del Covid-19. Mediatizzazione, liveness e pubblici, in «Mediascapes Journal», n. 15, 2020, pp. 44-58.

D. Harvey, The Right to The City, in «New Left Review», vol. 53, 2008, (ultimo accesso 23/11/2021).

M. Hardt, A. Negri, Comune. Oltre il privato e il pubblico, Rizzoli, Milano 2010.

U. Mattei, Beni Comuni. Un Manifesto, Laterza, Bari 2011.

Melucci A., New Social Movements. A theoretical Approach, in "Social Science Information", vol. 19, n. 2, 1980, pp. 199-226.

A. Moore, Occupation Culture: Art \& Squatting in the City from Below, Autonomedia, Brooklyn 2015.

C. Mouffe, P. Holdengräber, Radical Democracy: Modern or Postmodern?, in «Social Text», vol. 21, 1989, p. 41.

E. Ostrom, Governing the Commons, Cambridge University Press, Cambridge 1990.

L. Perelli, Public Art. Arte, interazione e progetto urbano, FrancoAngeli, Milano 2015.

$\mathrm{H}$. Prujit, The Logic of Urban Squatting, in «International Journal of Urban and Regional Research», vol. 37, n. 1, 2011 (ultimo accesso 23/11/2021).

J. Ranciére, The Emancipated Spectator, Verso Books, London \& New York 2009.

G. Raunig, G. Ray (a cura di), Art and contemporary critical practice: reinventing institutional critique, MayFly Books, London 2009.

P. Scannell, Authenticity and Experience, in «Discourse Studies», vol. 3, n. 4, 2001, pp- 405-411.

C. Tilly, Contentious Repertoires in Great Britain, 1758-1834, in "Social Science History», vol. 17 , n. 2, 1993, pp. 253-280.

J.L. Thompson, The World of the Social Entrepreneur, in «International Journal of Public Sector Management», vol. 15, n. 5, 2002.

M.P. Vittoria, P. Napolitano, Comunità informali come luoghi creativi e drivers di produttività urbana. Il caso dei Centri Sociali a Napoli, in "Rivista economica del Mezzogiorno», Fascicolo 1-2, 2017.

\section{Biografia dell'autore/ Author's biography}

Sara Pastore è dottoranda presso il Dipartimento di Scienze Sociali e Statistiche dell'Università Federico II di Napoli, dove si occupa di studi al crocevia tra gli ambiti della mediologia, della sociologia dell'arte e della museologia. Attualmente conduce un progetto di ricerca che si configura come uno studio esplorativo sul movimento alteristituzionale in Italia, focalizzando sulle alteristituzioni culturali 
autonome: quegli spazi occupati che si offrono come laboratori e cantieri per la produzione, la divulgazione e la fruizione dell'arte, nonché come luoghi di contestazione e sperimentazione metodologica istituzionale e civica.

Sara Pastore is a PhD candidate at the Social Sciences and Statistics Department of University of Naples "Federico II", where she is currently carrying out studies in the fields of media studies, sociology of art and museology. She is now conducting a research project which aims to explore the alterinstitutional movement in Italy, focusing on the autonomous cultural alterinstitutions: those occupied spaces that offer themselves as laboratories for the production, dissemination and enjoyment of art, as well as places of institutional and civic contestation and methodological experimentation.

Articolo sottoposto a double-blind peer review 